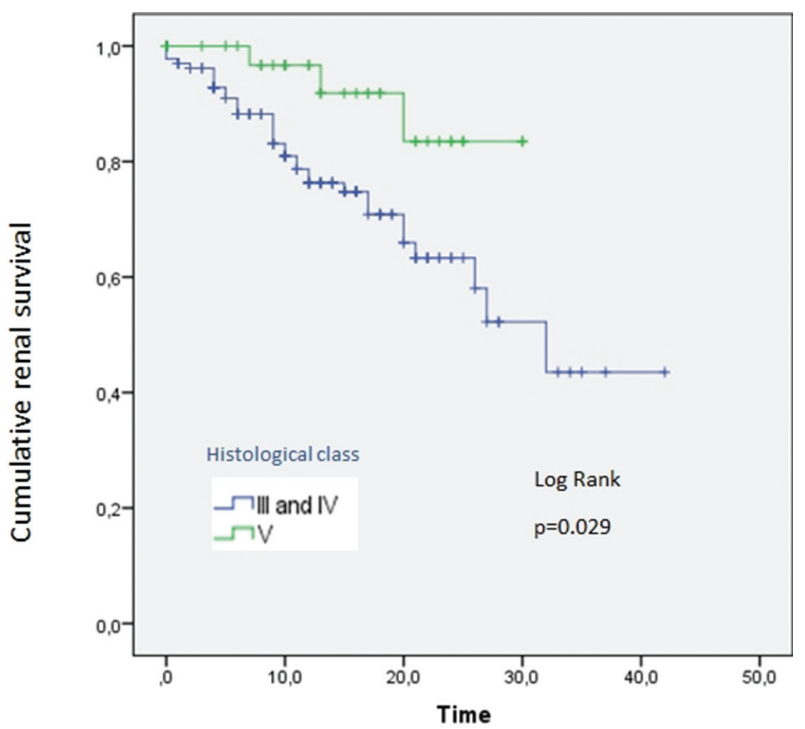

Conclusions: In spite of presenting in the context of the same autoimmune systemic disease, PLN and MLN appear to be very different entities, showing significant differences regarding serologic profiles and renal survival.

Disclosure of Interest: None declared

DOI: 10.1136/annrheumdis-2018-eular.5433

\section{SAT0433 APPLICATION OF THE DORIS ALGORITHM FOR THE DEFINITION OF DISEASE REMISSION OVER A 2-YEAR PERIOD IN A COHORT OF ITALIAN PATIENTS WITH SYSTEMIC LUPUS ERYTHEMATOSUS}

F. Dall'ara ${ }^{1}$, L. Andreaoli ${ }^{1}$, G. Armentaro ${ }^{1}$, F. Migliorati $^{1}$, M. Frassi $^{1}$, F. Franceschini ${ }^{1}$, S. Calza ${ }^{2}$, A. Tincani ${ }^{1} .{ }^{1}$ Rheumatology and Clinical Immunology, Department of Clinical and Experimental Sciences, Spedali Civili and University of Brescia; ${ }^{2}$ Department of Translational and Molecular Medicine, Biostatistics Unit, University of Brescia, Brescia, Italy

Background: Systemic Lupus Erythematosus (SLE) is characterised by a fluctuating course. To achieve sustained remission is the ultimate goal of maintenance treatment. However remission is difficult to define in SLE. In 2014, an international Task Force named DORIS proposed four definitions of remission. ${ }^{1}$

Objectives: Aim of this study was to evaluate the performance of the DORIS algorithm in comparison to the remission status as defined by clinical judgement and to identify the frequency of remission as determined by DORIS for each clinical disease pattern.

Methods: Monocentric retrospective study. Among all SLE patients followed at the Lupus Clinic between 2014 and 2016, we enrolled patients fulfilling the SLICC 2012 criteria who were visited at least once in 2016 and who had at least 5 biannual medical examinations in the previous 2 years. Definitions of remission according to DORIS, Clinical Remission, and disease patterns are reported in table 1.

Results: 101 SLE patients were enrolled for this study (94\% female, mean age 45 years). $17.8 \%$ of patients were in remission in all the 5 time-points, vice versa $29.7 \%$ of patients never got into remission. $17.8 \%$ of patients have been in remission for 24 months, while $21.8 \%$ of patients less then 6 months. Mean duration of DORIS remission was 7.96 months. The most prevalent disease patter were RR $(41,6 \%)$ and CQ $(41,6 \%)$, while CA pattern was present in $16,8 \%$ of patients. DORIS remission was most frequently achieved in CQ pattern (65.2\% of visits), less frequently in CA (5.9\%). 294 visits out of $505(58 \%)$ were defined as "nonremission" according to DORIS. cSLEDAl above zero was the item that most frequently accounted for "non-remission", particularly urinary and haematological (as reported in figure 1). In 229 (43.3\%) visits there was a disagreement between DORIS and clinical judgement: the reasons for discordant results were respectively: a) self-management of steroids dosage and precautionary increase of steroids in the suspect of a flare in $7.1 \%$; b) cSLEDAI $>0$ in $27.2 \%, P G A \geq 0.5$ in $12.6 \%$, more than one of these items in $53.1 \%$.
Abstract SAT0433 - Table 1. Definition of Remission according to Doris, Definition of Clinical Remission according to Clinical judgement; Definition of disease pattern. PGA: Physician Global Assessment; cSLEDAI: clinical SLEDAI

\begin{tabular}{|c|c|c|c|c|c|}
\hline \multirow[t]{2}{*}{ Definition of Remi ssion } & \multicolumn{2}{|c|}{ Clinical Items } & \multicolumn{3}{|c|}{ Treatment } \\
\hline & CSLEDAI & PGA & $\begin{array}{l}\text { Daily dose of } \\
\text { prednisone }\end{array}$ & Antimalarials & $\begin{array}{c}\text { Immunosuppressants/ } \\
\text { Biologics }\end{array}$ \\
\hline Remission "off treatment" & $=0$ & $<0.5$ & 0 & yes & no \\
\hline Remission "on treatment" & $=0$ & $<0.5$ & $\leq 5 \mathrm{mg}$ & yes & yes \\
\hline No Remission & $\neq 0$ & $\geq 0.5$ & $25 \mathrm{mg}$ & yes & yes \\
\hline $\begin{array}{l}\text { Definition of Clinical judgement } \\
\text { of Remission }\end{array}$ & \multicolumn{5}{|c|}{$\begin{array}{l}\text { Ab sence of any increase in corticosteroids dosage or any change in } \\
\text { immunosuppressants }\end{array}$} \\
\hline \multicolumn{6}{|l|}{ Definition of disease patterns } \\
\hline Chronic active (CA) & \multicolumn{5}{|c|}{$\begin{array}{l}\text { Persistent disease activity over time with a cSLEDAI } 11 \text { in each visit for at least } \\
\text { one year }\end{array}$} \\
\hline Relapsing-remitting (RR) & \multicolumn{5}{|c|}{$\begin{array}{l}\text { Characterised by periods of disease activity with CLLEDAl } \geq 1 \text { interspers ed with } \\
\text { periods of disease inactivity with cSLEDAl = } 0 \text { in different visits for at least one } \\
\text { year }\end{array}$} \\
\hline Clinical quiescent (CQ) & \multicolumn{5}{|c|}{ Defined as absence of disease activity with a cSLEDAl $=0$ for at least one year } \\
\hline
\end{tabular}

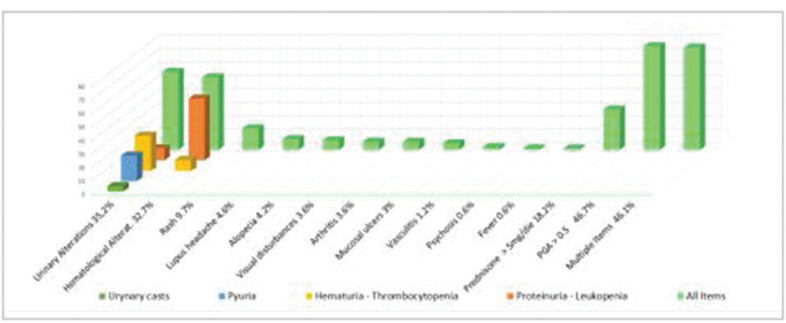

Abstract SAT0433 - Figure 1. Items are responsible of disagreement between DORIS and Clinical definition of Remission

Conclusions: Nearly $40 \%$ of the visits displayed a disagreement between clinical judgement of remission and DORIS remission. This may be attributable mainly to a different approach in evaluating patients: longitudinal by clinical judgement and cross-sectional by DORIS. As compared to clinical judgement of remission, the DORIS definition is not designed to capture "low disease activity", particularly patients who carry a PGA between 0.5 and 1 and those who require a medium dosage of steroids in the frame of a CA pattern.

\section{REFERENCE:}

[1] van Vollenhoven R, et al. Ann Rheum Dis 2017 Mar;76(3):554-561.

Disclosure of Interest: None declared

DOI: 10.1136/annrheumdis-2018-eular.6480

\section{SAT0434 \\ PARTICIPATION IN SYSTEMIC LUPUS ERYTHEMATOSUS - A CROSS-SECTIONAL ANALYSIS OF THE LULA-COHORT IN GERMANY 2015}

G. Chehab $^{1}$, J.G. Richter ${ }^{1}$, R. Fischer-Betz ${ }^{1}$, R. Brinks ${ }^{1}$, B. Winkler-Rohlfing ${ }^{2}$, M. Schneider'. ${ }^{1}$ Policlinic of Rheumatology and Hiller Research Unit Rheumatology, Medical Faculty, Heinrich-Heine-University, Duesseldorf, ${ }^{2}$ German Lupus Self-Help Community, Wuppertal, Germany

Background: Participation of chronically ill patients can be permanently and severely impaired. In a heterogeneous disease like systemic lupus erythematosus (SLE), various influencing factors such as disease activity, damage, concomitant diseases, but also detrimental effects due to the psychological burden must be considered.

Objectives: Our objective was to assess the current state of participation in a representative sample of German patients with SLE and evaluate the impact of demographic and clinical factors.

Methods: The Lupus erythematosus long-term study (LuLa-Study), a nationwide longitudinal study among German Caucasian patients with SLE, is being 